\title{
INFINITELY DIVISIBLE POSITIVE DEFINITE SEQUENCES
}

\author{
BY
}

ROGER A. HORN( $\left.{ }^{1}\right)$

Introduction. Sequences of complex numbers which generate positive semidefinite Toeplitz or Hankel matrices occur naturally in many areas of mathematics and are usually called positive definite sequences. It is well known that a sequence formed from a positive integer power of the terms of a positive definite sequence is always itself a positive definite sequence, but we shall be interested in the sequences of the title, which have the special property that any sequence formed from a positive fractional power of their terms is again a positive definite sequence. These sequences will be characterized and representation formulae will be derived which relate them to certain moment sequences. Their relationship with classical conformal mapping problems will be discussed and the natural interpolation problems for them will be solved.

We shall use without further comment the methods and the notation of [5]. As a natural extension of that notation, if $A \equiv\left(a_{i j}\right)_{i, j=0}^{\infty}$ is an infinite matrix we shall write $A \succeq 0$ (positive semidefinite) or $A \succeq 0$ on $L$ if and only if every finite section $A_{N} \equiv\left(a_{i j}\right)_{i, j=0}^{N}, \quad N=0,1,2, \ldots$, satisfies $A_{N} \succeq 0$ or $A_{N} \succeq 0$ on $L^{N+1} \equiv$ $\left\{x \in C^{N+1} \mid \sum_{i=1}^{N+1} x_{i}=0\right\}$, respectively.

1. Toeplitz forms. If $\left\{a_{n}\right\}_{-\infty}^{\infty} \subset \boldsymbol{C}$ is a sequence of complex numbers, we shall say that $\left\{a_{n}\right\}_{-\infty}^{\infty}$ is a positive definite $(-)$ sequence and write $\left\{a_{n}\right\}_{n=-\infty}^{\infty} \geq 0(-)$ if the Toeplitz matrix $A \equiv\left(a_{i-j}\right)_{i, j=0}^{\infty} \succeq 0$. Such sequences have played an important role in analysis ever since they appeared circa 1910 in fundamental works of Carathéodory, Toeplitz, Fejér, Fischer, and Herglotz on analytic functions with positive real part in the unit disc. We state first some of the well-known (vid. [3, p. 35]) fundamental properties of positive definite $(-)$ sequences as

THEOREM 1.1. Let $\left\{a_{n}\right\}_{-\infty}^{\infty} \geq 0(-)$. Then (a) $a_{0} \geqq 0$; (b) $a_{-n}=\bar{a}_{n}$ for all $n=0,1, \ldots$; (c) $\left|a_{n}\right| \leqq a_{0}$ for all $n=0,1,2, \ldots$; (d) if $\left\{b_{n}\right\}_{n=-\infty}^{\infty} \geq 0(-)$, then $\left\{a_{n} b_{n}\right\}_{-\infty}^{\infty} \geq 0(-)$; and (e) if for some $0 \leqq \theta<2 \pi$ and for some $N \geqq 1, a_{N}=e^{i \theta} a_{0}$, then $a_{n}=\exp (i[[n / N]] \theta) a_{n \bmod N}$ for all $n=0,1,2, \ldots$, where $[[n / N]] \equiv$ greatest integer in $n / N$.

We see, therefore, that there will be no loss of generality to assume in all that follows that $a_{0}=1$ and that we may restrict our attention to $\left\{a_{n}\right\}_{n=0}^{\infty}$ if we agree that $a_{-n} \equiv \bar{a}_{n}, \arg a_{-n} \equiv-\arg a_{n}$. Much of the usefulness of positive definite (-)

Received by the editors August 21, 1957.

( $\left.{ }^{1}\right)$ This work was supported in part by National Science Foundation Grant GP 7656 and by the U.S. Army Research Office DA-31-124-ARO(D)-151. 
sequences stems from their connection with the trigonometric moment problem and the existence of a simple representation formula for them.

THEOREM $1.2\left[1\right.$, pp. 508-511]. Let $\left\{a_{n}\right\}_{-\infty}^{\infty} \subset C$. Then $\left\{a_{n}\right\}_{n=-\infty}^{\infty} \geq 0(-), a_{0}=1$, if and only if

$$
a_{n}=\int_{-\pi}^{\pi} e^{i n \theta} d \mu(\theta), \quad-\infty<n<+\infty
$$

for a unique probability measure $d \mu$, i.e., $d \mu \geqq 0$ and $\int_{-\pi}^{\pi} d \mu(\theta)=1$.

We shall be interested primarily in those positive definite $(-)$ sequences which are infinitely divisible according to the

Definition. Let $\left\{a_{n}\right\}_{n=-\infty}^{\infty} \succeq 0(-), a_{0}=1$, i.e., $A=\left(a_{i-j}\right)_{i, j=0}^{\infty} \geq 0$, and let some choice of arguments be made, $\arg a_{-n} \equiv-\arg a_{n}$ for all $n=0,1,2, \ldots$ Then $\left\{a_{n}\right\}_{n=0}^{\infty}$ is said to be infinitely divisible if and only if $A$ is infinitely divisible with this choice of arguments, i.e., if and only if $A^{\alpha} \equiv\left(a_{i-j}^{\alpha}\right)_{i, j=0}^{\alpha} \succeq 0$ for all $\alpha>0$.

Thus, $\left\{a_{n}\right\}_{n=0}^{\infty} \geq 0(-)$ is infinitely divisible if and only if with some choice of arguments (which will be held fixed throughout) $\left\{a_{n}^{\alpha}\right\}_{n=0}^{\infty} \geq 0(-)$ for all $\alpha>0$, while this is the case if and only if for each $\alpha>0$ there is a (unique) probability measure $d \mu_{\alpha}$ such that $a_{n}^{\alpha}=\int_{-\pi}^{\pi} e^{i n \theta} d \mu_{\alpha}(\theta)$ for all $n=0,1,2, \ldots$ Let $N \geqq 1$ be an integer, suppose $\left\{a_{n}\right\}_{n=0}^{\infty}$ is infinitely divisible, and notice that for every $n=0,1, \ldots$,

$a_{n}=\left(a_{n}^{1 / N}\right)^{N}=\left(\int_{-\pi}^{\pi} e^{i n \theta} d \mu_{1 / N}(\theta)\right)^{N}=\int_{-\pi}^{\pi} e^{i n \theta}\left(d \mu_{1 / N} * \cdots * d \mu_{1 / N}\right)(\theta)=\int_{-\pi}^{\pi} e^{i n \theta} d \mu(\theta)$, ( $N$ times)

where by $(d \mu * d \nu)(\theta)$ we mean the additive convolution mod $2 \pi$ of measures, i.e., convolution in the circle group. By the uniqueness statement in Theorem 1.2, we see that for each $N=1,2,3, \ldots$ the probability measure $d \mu_{1 / N}$ is such that

$$
d \mu(\theta)=\left(d \mu_{1 / N} * \cdots * d \mu_{1 / N}\right)(\theta) \equiv\left(d \mu_{1 / N}\right)^{* N}(\theta)
$$

( $N$ times)

Furthermore, the family of probability measures $\left\{d \mu_{t}\right\}_{t>0}$ has the property that $d \mu_{s} * d \mu_{t}=d \mu_{s+t}$ for all $s, t>0$. We formalize these observations in the

DEFINITION. Let $d \mu$ be a nonnegative measure on a locally compact semigroup $G$ and let $*$ denote the convolution operation between measures on $G$. Then $d \mu$ is said to be infinitely divisible if and only if for every positive integer $n$ there exists a nonnegative measure $d \mu_{n}$ such that $d \mu=\left(d \mu_{n}\right)^{* n}$. We shall say that $d \mu$ is strongly infinitely divisible (imbeddable) if and only if there exists a continuous family $\left\{d \mu_{t}\right\}_{t} \geq_{0}$ of nonnegative measures such that $d \mu_{1}=d \mu$ and $d \mu_{s} * d \mu_{t}=d \mu_{s+t}$ for all $s, t \geqq 0$.

REMARK. It is well known that if $G=\boldsymbol{R}$ (under addition), then every infinitely divisible measure is strongly infinitely divisible. More generally, it is known that this is also true if $G$ is any locally compact Abelian group whose dual group is arcwise connected. We shall have another example of this phenomenon below. 
A probability measure $d \mu$ on the circle group is strongly infinitely divisible if and only if the positive definite (-) sequence $\left\{\int_{-\pi}^{\pi} e^{i n \theta} d \mu\right\}_{n=0}^{\infty}$ is infinitely divisible, and so we shall be able to obtain information about such measures by studying infinitely divisible positive definite sequences. Nearly all our results can be established directly from properties of quadratic forms independent of the measures themselves.

THEOREM 1.3. Let $\left\{a_{n}\right\}_{n=0}^{\infty} \succeq 0(-), a_{0}=1$, be infinitely divisible. If $a_{1} \neq 0$, then all $a_{n} \neq 0, n=1,2,3, \ldots$ If $a_{1}=0$, then either all $a_{n}=0, n=1,2,3, \ldots$, or there exists an integer $N, 2 \leqq N<\infty$, such that $a_{n} \neq 0$ if and only if $n \equiv 0(\bmod N)$, $n=1,2,3, \ldots$ These statements are mutually exclusive and exhaust all possibilities.

Proof. If the matrix $A=\left(a_{i-j}\right)_{i, j=0}^{\infty}$ is infinitely divisible, then its incidence matrix $M(A)=\left(\mu_{i-j}\right)_{i, j=0}^{\infty} \succeq 0$, where

$$
\begin{aligned}
\mu_{n} \equiv 1 & \text { if } a_{n} \neq 0 \\
\equiv 0 & \text { if } a_{n}=0
\end{aligned}
$$

By [5, Theorem 1.13] it is necessary and sufficient for this that every principal third-order submatrix of the incidence matrix generates a nonnegative quadratic form, i.e.,

$$
\left(\begin{array}{lll}
\mu_{0} & \mu_{i-j} & \mu_{i-k} \\
\mu_{i-j} & \mu_{0} & \mu_{j-k} \\
\mu_{i-k} & \mu_{j-k} & \mu_{0}
\end{array}\right) \geq 0, \quad \text { for all } i>j>k \geqq 0 .
$$

Suppose $a_{1} \neq 0$ and let $k=0, j=1, i=n+1, n \geqq 1$, so that

$$
\left(\begin{array}{lll}
\mu_{0} & \mu_{n} & \mu_{n+1} \\
\mu_{n} & \mu_{0} & \mu_{1} \\
\mu_{n+1} & \mu_{1} & \mu_{0}
\end{array}\right)=\left(\begin{array}{lll}
1 & \mu_{n} & \mu_{n+1} \\
\mu_{n} & 1 & 1 \\
\mu_{n+1} & 1 & 1
\end{array}\right) \geq 0,
$$

and consequently $\mu_{n} \neq 0$ implies $\mu_{n+1} \neq 0$ for all $n=1,2,3, \ldots$ Hence all $a_{n} \neq 0$. Now suppose $a_{1}=0$ but that not all $a_{n} \equiv 0, n \geqq 1$. Let $\infty>N \equiv \min \left\{n \mid a_{n} \neq 0\right\} \geqq 2$ and let $k=0, j=N, i=N+n, n \geqq 1$ so that by (1.A) again

$$
\left(\begin{array}{lll}
\mu_{0} & \mu_{n} & \mu_{N+n} \\
\mu_{n} & \mu_{0} & \mu_{N} \\
\mu_{N+n} & \mu_{N} & \mu_{0}
\end{array}\right)=\left(\begin{array}{lll}
1 & \mu_{n} & \mu_{N+n} \\
\mu_{n} & 1 & 1 \\
\mu_{N+n} & 1 & 1
\end{array}\right) \geq 0,
$$

and consequently $\mu_{n}=0$ implies $\mu_{N+n}=0$ and $\mu_{n} \neq 0$ implies $\mu_{N+n} \neq 0$. But $\mu_{n}=0$ for all $1 \leqq n \leqq N-1$ so $\mu_{n}=0$ if $n \not \equiv 0(\bmod N)$. Finally, $\mu_{N} \neq 0$, so $\mu_{n} \neq 0$ if $n \equiv$ $0(\bmod N)$. Q.E.D.

Definition. If $d \mu(\theta)$ is a probability measure on the unit circle $\left\{e^{i \theta} \mid-\pi \leqq \theta<\pi\right\}$, define

$$
C(d \mu) \equiv \int_{-\pi}^{\pi} e^{i \theta} d \mu(\theta)
$$


$C(d \mu)$ is the mass center or first moment of the measure $d \mu$. The $n$th moment of $d \mu$ is

$$
a_{n} \equiv \int_{-\pi}^{\pi} e^{i n \theta} d \mu(\theta), \quad n=2,3,4, \ldots
$$

In this language we have immediately the

COROLlARY 1.4. Let $d \mu(\theta)$ be a strongly infinitely divisible probability measure on the unit circle. Then either the mass center of $d \mu$ is at zero or not. If not, then no higher moment of $d \mu$ can vanish. If the mass center is at zero, then either $d \mu(\theta)$ is the normalized Lebesgue measure on the unit circle, i.e., $d \mu(\theta)=d \theta / 2 \pi$, or there exists an integer $2 \leqq N<\infty$ such that for each of the higher moments $a_{n}, a_{n}=0$ if and only if $n \not \equiv 0(\bmod N)$. These statements are mutually exclusive and exhaust all possibilities.

We shall exploit the fact that the zeros, if any, in an infinitely divisible positive definite $(-)$ sequence $\left\{a_{n}\right\}_{n=0}^{\infty}$ must occur with a rigid regularity. Because of this and the following proposition, there will be no loss of generality when we assume in what follows that all $a_{n} \neq 0$.

Definition. If $\left\{a_{n}\right\}_{n=0}^{\infty} \subset C$ we define the $k$-expansion operator $E_{k}\left\{a_{n}\right\}_{n=0}^{\infty} \equiv$ $\left\{b_{n}^{(k)}\right\}_{n=0}^{\infty}$ for any $k=1,2,3, \ldots$ by

$$
\begin{array}{rlrl}
b_{n}^{(k)} & \equiv a_{m} & \text { if } n=k m \\
& \equiv 0 & & \text { otherwise. }
\end{array}
$$

We shall call the sequence $\left\{a_{n}\right\}_{n=0}^{\infty}$ a $k$-sequence if and only if $1 \leqq k \leqq \infty$ and either $\left\{a_{n}\right\}_{n=0}^{\infty}=\left\{a_{0} \delta_{n 0}\right\}_{n=0}^{\infty}$ if $k=\infty$, or $\left\{a_{n}\right\}_{n=0}^{\infty}=E_{k}\left\{c_{n}\right\}_{n=0}^{\infty}$ if $k<\infty$, where all $c_{n} \neq 0$. If $k<\infty$, this (unique) sequence $\left\{c_{n}\right\}_{n=0}^{\infty}$ is called the fundamental sequence of $\left\{a_{n}\right\}_{n=0}^{\infty}$.

Proposition 1.5. Let $k \geqq 1$. Then $\left\{a_{n}\right\}_{n=0}^{\infty} \geq 0(-)$ if and only if $E_{k}\left\{a_{n}\right\}_{n=0}^{\infty} \geq 0(-)$.

Proof. If we consider the identity

$$
\left\langle x, B^{(k)} x\right\rangle=\sum_{i, j=0} x_{i} b_{i-j}^{(k)} \bar{x}_{j}=\sum_{l=0}^{k-1}\left\langle x^{(k, l)}, A x^{(k, l)}\right\rangle
$$

where $\left\{b_{n}^{(k)}\right\}_{n=0}^{\infty} \equiv E_{k}\left\{a_{n}\right\}_{n=0}^{\infty}, B^{(k)} \equiv\left(b_{i-j}^{(k)}\right)_{i, j=0}^{\infty}$ and $x^{(k, l)} \equiv\left(x_{i}^{(k, l)}\right)_{i=0}^{\infty}=\left(x_{l+i k}\right)_{i=0}^{\infty}$, we see that $B^{(k)} \geq 0$ if and only if $A \geq 0$. Q.E.D.

COROllary 1.6. The sequence $\left\{a_{n}\right\}_{n=0}^{\infty}, a_{0}=1$, is an infinitely divisible positive definite sequence if and only if either $\left\{a_{n}\right\}_{n=0}^{\infty}=\left\{\delta_{n 0}\right\}_{n=0}^{\infty}$ or, for some $1 \leqq k<\infty$, $\left\{a_{n}\right\}_{n=0}^{\infty}$ is a $k$-sequence whose fundamental sequence is an infinitely divisible positive definite (-) sequence.

By a direct quotation of [5, Corollary 1.6] we now have the basic characterization contained in

THEOREM 1.7. Let $\left\{a_{n}\right\}_{-\infty}^{\infty} \subset C, a_{0}=1$. Then $\left\{a_{n}\right\}_{-\infty}^{\infty}$ is an infinitely divisible positive definite (-) sequence if and only if (a) $\left\{a_{n}\right\}_{n=0}^{\infty}$ is a $k$-sequence for some $1 \leqq k \leqq \infty$, (b) $a_{-n}=\bar{a}_{n}$ for all $n=0,1,2, \ldots$, and (c) if $k<\infty$, the fundamental sequence 
$\left\{c_{n}\right\}_{n=0}^{\infty},\left\{a_{n}\right\}_{n=0}^{\infty}=E_{k}\left\{c_{n}\right\}_{n=0}^{\infty}$, has the property that $B \equiv\left(b_{i-j}\right)_{i, j=0}^{\infty} \equiv\left(\log c_{i-j}\right)_{i, j=0}^{\infty} \succeq 0$ on $L$ for some choice of arguments, where we take $c_{-n} \equiv \bar{c}_{n}, \arg c_{-n} \equiv-\arg c_{n}$, $n=0,1,2, \ldots$

This theorem, then, provides effective necessary and sufficient conditions that a probability measure on $[-\pi, \pi]$ be strongly infinitely divisible. Before deriving a representation formula for all infinitely divisible positive definite $(-)$ sequences, we shall study sequences which satisfy the positivity condition in part (c) above.

THEOREM 1.8. Let $\left\{b_{n}\right\}_{-\infty}^{\infty} \subset C, b_{0}=0$, satisfy the two conditions (i) $\operatorname{Re} b_{-n}=\operatorname{Re} b_{n}$ for all $n=0,1,2, \ldots$, and (ii) $B \equiv\left(b_{i-j}\right)_{i, j=0}^{\infty} \geq 0$ on L. Then (a) $\operatorname{Re} b_{n} \leqq 0$ for all $n=0, \pm 1, \pm 2, \ldots$; (b) $b_{-n}=\bar{b}_{n}$ for all $n=0,1,2, \ldots$; (c) if $\operatorname{Re}\left(b_{K}\right)=0$ for some $K$, $1 \leqq K<\infty$, then $b_{n}=b_{n \bmod K}+\left[\left[n K^{-1}\right]\right] b_{K}$ for all $n=1,2, \ldots ;$ (d) $\left|b_{k n}\right| \leqq n^{2}\left|b_{k}\right|$ for all $k, n=1,2,3, \ldots ;$ and (e) in particular, $\left|b_{n}\right| \leqq n^{2}\left|b_{1}\right|$ for all $n=0,1,2, \ldots$.

Proof. By [5, Theorem 1.10],

$$
\left(\begin{array}{ll}
b_{0} & b_{n} \\
b_{-n} & b_{0}
\end{array}\right) \geq 0
$$

on $L^{2}$ if and only if $b_{0}+b_{0}-b_{n}-b_{-n}=-2 \operatorname{Re} b_{n}-i \operatorname{Im}\left(b_{n}+b_{-n}\right) \geqq 0$. But then $\operatorname{Re} b_{n} \leqq 0, \operatorname{Im} b_{n}=-\operatorname{Im} b_{-n}$, and $b_{-n}=\bar{b}_{n}$, so (a) and (b) are proved.

To prove (c), let $m>n>0$ and use [5, Theorem 1.10] again to show that

$$
\left(\begin{array}{lll}
b_{0} & b_{n} & b_{m} \\
\bar{b}_{n} & b_{0} & b_{m-n} \\
\bar{b}_{m} & \bar{b}_{m-n} & b_{0}
\end{array}\right)=\left(\begin{array}{lll}
0 & b_{n} & b_{m} \\
\bar{b}_{n} & 0 & b_{m-n} \\
\bar{b}_{m} & \bar{b}_{m-n} & 0
\end{array}\right) \geq 0 \quad \text { on } L^{3}
$$

if and only if

$$
\left(\begin{array}{cc}
-2 \operatorname{Re} b_{n} & b_{n}+b_{m-n}-b_{m} \\
\bar{b}_{n}+\bar{b}_{m-n}-\bar{b}_{m} & -2 \operatorname{Re} b_{m-n}
\end{array}\right) \succeq 0 .
$$

But then the determinant must be nonnegative, so

$$
4\left(\operatorname{Re} b_{n}\right)\left(\operatorname{Re} b_{m-n}\right) \geqq\left|b_{n}+b_{m-n}-b_{m}\right|^{2} .
$$

If $\operatorname{Re}\left(b_{K}\right)=0$, let $n=K$ in this inequality to conclude that $\left|b_{K}+b_{m-K}-b_{m}\right| \equiv 0$, i.e., $b_{m}=b_{m \bmod K}+\left[\left[m K^{-1}\right]\right] b_{K}, m=K+1, K+2, \ldots$

For part (d), observe that the desired inequality $\left|b_{k n}\right| \leqq n^{2}\left|b_{k}\right|$ is true for all $k$ for $n=1$. Assume inductively that it is true for all $k$ for $n=1,2, \ldots, l-1, l \geqq 2$, and prove it must be true for $n=l$ as follows: Take $m=l k, n=k$ in (1.B), so that

$$
\left|b_{k}+b_{(l-1) k}-b_{l k}\right|^{2} \leqq 4\left(\operatorname{Re} b_{k}\right)\left(\operatorname{Re} b_{(l-1) k}\right) \leqq 4\left|b_{k}\right|\left|b_{(l-1) k}\right| \leqq 4(l-1)^{2}\left|b_{k}\right|^{2}
$$

by the induction hypothesis, i.e.,

$$
\left|b_{k}+b_{(l-1) k}-b_{l k}\right| \leqq 2(l-1)\left|b_{k}\right| .
$$


But then

$$
2(l-1)\left|b_{k}\right| \geqq\left|b_{l k}\right|-\left|b_{k}\right|-\left|b_{(l-1) k}\right| \geqq\left|b_{l k}\right|-\left|b_{k}\right|-(l-1)^{2}\left|b_{k}\right|
$$

so that

$$
l^{2}\left|b_{k}\right| \geqq\left|b_{l k}\right|
$$

as desired. Q.E.D.

Although we have derived them directly, parts (a), (b), and (c) could have been deduced from Theorem 1.7 and the corresponding already known properties of infinitely divisible positive definite $(-)$ sequences. The growth estimate of part (d) is a new fact, however, and shows that the terms of an infinitely divisible positive definite ( - ) sequence $\left\{a_{n}\right\}_{n=0}^{\infty}$ with $a_{0}=1, a_{1} \neq 0$ cannot tend to zero arbitrarily fast, as is possible with the terms of a sequence which is merely positive definite $(-)$. This may be expressed precisely in the form of the inequality $\left|a_{n}\right| \geqq \exp \left(-n^{2}\left|\log a_{1}\right|\right)$, $n \geqq 1$, which holds even if $a_{1}=0$. Since $\left\{\exp \left[-n^{2}\right]\right\}_{n=0}^{\infty} \geq 0(-)$ is infinitely divisible, the inequality is sharp.

We now give the representation formula for infinitely divisible sequences analogous to the Herglotz representation of Theorem 1.2.

THEOREM 1.9. Let $\left\{a_{n}\right\}_{n=-\infty}^{\infty} \subset C, a_{0}=1, a_{1} \neq 0$. Then $\left\{a_{n}\right\}_{n=-\infty}^{\infty}$ is an infinitely divisible positive definite (-) sequence if and only if $a_{-n}=\bar{a}_{n}$ and there exists a (unique) nonnegative measure $d \mu$ on $[-\pi, \pi)$ with $\int_{-\pi}^{\pi} d \mu(\theta)=-2 \operatorname{Re} \log a_{1}$ such that for all $n=0,1,2,3, \ldots, a_{n}=e^{b_{n}}$, where

$$
b_{n}=n \log a_{1}-\int_{-\pi}^{\pi} \frac{e^{i(n-1) \theta}+(n-1) e^{-i \theta}-n}{\left(1-e^{-i \theta}\right)^{2}} d \mu(\theta) .
$$

Proof. The sufficiency of the conditions is apparent from Theorem 1.7, for if $x \in L^{N+1}$, then $\sum_{n=0}^{N} x_{n}=0$ and

$$
\infty>\sum_{i, j=0}^{N} x_{i} b_{i-j} \bar{x}_{j}=\frac{1}{4} \int_{-\pi}^{\pi}\left|\sum_{n=0}^{N} e^{i n \theta} x_{n}\right|^{2} \frac{d \mu(\theta)}{\sin ^{2}(\theta / 2)} \geqq 0 .
$$

Notice that all $a_{n} \neq 0$ since all $b_{n}$ are finite.

For the necessity, we know that if $\left\{a_{n}\right\}_{n=0}^{\infty}$ is infinitely divisible with $a_{0}=1$, $a_{1} \neq 0$, then all $a_{n} \neq 0$. Theorem 1.7 and [5, Theorem 1.10$]$ imply that the new sequence

$$
\left\{\log a_{n}+\log a_{n}-\log a_{n+1}-\log a_{n-1}\right\}=\left\{\log \left(a_{n}^{2} / a_{n+1} a_{n-1}\right)\right\}_{n=0}^{\infty} \geq 0(-),
$$

and so by Theorem 1.2 there exists a (unique) nonnegative measure $d \mu(\theta)$ on $[-\pi, \pi)$,

such that

$$
\int_{-\pi}^{\pi} d \mu(\theta)=\log \frac{a_{0}^{2}}{a_{1} a_{-1}}=\log \frac{1}{\left|a_{1}\right|^{2}}=-2 \operatorname{Re} \log a_{1},
$$

$$
\log \frac{a_{n}^{2}}{a_{n+1} a_{n-1}}=\int_{-\pi}^{\pi} e^{i n \theta} d \mu(\theta), \quad n=0,1,2, \ldots
$$


This recurrence relation is easily solved for $\log a_{n}$. Set $c_{n} \equiv a_{n}^{2}\left(a_{n+1} a_{n-1}\right)^{-1}, n \geqq 1$, so that $a_{n+1}=a_{1}^{n+1}\left(a_{0} c_{n} c_{n-1}^{2} \cdots c_{2}^{n-1} c_{1}^{n}\right)^{-1}$, i.e.,

$$
\begin{aligned}
\log a_{n+1} & =(n+1) \log a_{1}-\sum_{k=1}^{n}(n-k+1) \log c_{k} \\
& =(n+1) \log a_{1}-\sum_{k=1}^{n}(n-k+1) \int_{-\pi}^{\pi} e^{i k \theta} d \mu(\theta) \\
& =(n+1) \log a_{1}-\int_{-\pi}^{\pi} \frac{e^{i n \theta}+n e^{-i \theta}-(n+1)}{\left(1-e^{-i \theta}\right)^{2}} d \mu(\theta), \quad n \geqq 1 .
\end{aligned}
$$

This is the announced formula, since it also holds for $n=-1,0$. Q.E.D.

As we should like to apply these results to the positive real part functions of classical function theory, we introduce some notation.

DEFINITION. The class of normalized positive real part functions will be denoted by $P \equiv\left\{f(z)\right.$ analytic for $\left.|z|<1 \mid f(0)=1, f^{\prime}(0) \geqq 0, \operatorname{Re} f(z) \geqq 0\right\}$.

If $f \in P$, then $f$ has a power series expansion convergent in the unit disc which we shall always write as $f(z)=1+2 \sum_{n=1}^{\infty} a_{n} z^{n}, a_{1} \geqq 0$.

DEFINITION. If $f(z)=1+2 \sum_{n=1}^{\infty} a_{n} z^{n} \in P$ and $g(z)=1+2 \sum_{n=1}^{\infty} b_{n} z^{n} \in P$, then $h(z) \equiv(f \circ g)(z)=(g \circ f)(z) \equiv 1+2 \sum_{n=1}^{\infty} a_{n} b_{n} z^{n}$ is the Hadamard product of $f$ and $g$.

THEOREM 1.10 [1, p. 502]. The function $f(z)=1+2 \sum_{n=1}^{\infty} a_{n} z^{n} \in P$ if and only if $a_{0}=1, a_{1} \geqq 0$ and $\left\{a_{n}\right\}_{n=0}^{\infty} \geq 0(-)$.

CoROLlaRY $1.11[1$, p. 511]. The function $f(z) \in P$ if and only if

$$
f(z)=\int_{-\pi}^{\pi} \frac{1+e^{i \theta} z}{1-e^{i \theta} z} d \mu(\theta)
$$

for some (unique) probability measure $d \mu(\theta)$ such that

$$
0 \leqq C(d \mu)=\int_{-\pi}^{\pi} e^{i \theta} d \mu(\theta) \leqq 1 .
$$

Proof. The above theorem and Theorem 1.2.

Corollary 1.12. If $f, g \in P$, then $f \circ g \in P$.

Proof. The above theorem and Theorem 1.1(d).

Definition. The function $f(z)=1+2 \sum_{n=1}^{\infty} a_{n} z^{n} \in P$ is said to be an infinitely divisible positive real part function if and only if $\left\{a_{n}\right\}_{n=0}^{\infty}, a_{0} \equiv 1$, is an infinitely divisible positive definite $(-)$ sequence. Let $P^{*}$ denote the class of infinitely divisible positive real part functions.

Thus, $f(z)=1+2 \sum_{n=1}^{\infty} a_{n} z^{n} \in P^{*}$ if and only if there exists a continuous family of functions $\left\{f_{\alpha}(z)\right\}_{\alpha \geqq 0} \subset P$ such that $f_{1}(z)=f(z)$ and $\left(f_{\alpha} \circ f_{\beta}\right)(z)=f_{\alpha+\beta}(z)$ for all $\alpha, \beta \geqq 0$. A direct translation of Theorem 1.3 in this case is the

TheOREM 1.13. Let $f(z) \in P^{*}$. If $f^{\prime}(0) \neq 0$, then all $f^{(n)}(0) \neq 0, n=1,2,3, \ldots$ If $f^{\prime}(0)=0$, then either $f(z) \equiv 1$ or for some (unique) $2 \leqq k<\infty, f(z) \equiv f\left(z \exp \left[i 2 \pi k^{-1}\right]\right)$ $\equiv f\left(z \exp \left[i 2 \pi k^{-1} 2\right]\right) \equiv \cdots \equiv f\left(z \exp \left[i 2 \pi k^{-1}(k-1)\right]\right)$, i.e., $f(z)$ is really a function of $z^{k}$, and $g_{k}(z) \equiv f\left(z^{1 / k}\right) \in P^{*}$ has $g_{k}^{\prime}(0) \neq 0$. 
The class $P^{*}$ consists then entirely of the constant function 1 , of functions none of whose coefficients vanishes and of functions $f(z)=g\left(z^{k}\right)$ where $g \in P^{*}$ has no vanishing coefficients and $k=2,3,4, \ldots$ Thus, $P^{*}$ is completely described by those of its elements $f(z)$ such that $f^{\prime}(0) \neq 0$. Since there are members of $P^{*}$ which are bounded, unbounded, schlicht, not schlicht, convex, not convex, etc., we do not have any simple geometrical characterization for $P^{*}$ of the kind we have for $P$. We can, however, provide a very simple characterization of the infinitesimal elements of $P^{*}$, i.e., of those functions $\Phi(z)$ such that

where $f \in P^{*}$.

$$
\left.2 \Phi(z) \equiv \frac{d}{d \alpha} f_{\alpha}(z)\right|_{\alpha=0} \equiv \frac{d}{d \alpha}\left[1+2 \sum_{n=1}^{\infty} a_{n}^{\alpha} z^{n}\right]_{\alpha=0},
$$

THEOREM 1.14. Let $f(z) \equiv 1+2 \sum_{n=1}^{\infty} a_{n} z^{n}$ be analytic in the unit disc, $f^{\prime}(0)>0$. Then $f \in P^{*}$ if and only if (a) all $a_{n} \neq 0$, (b) the infinitesimal generating function $\Phi(z) \equiv \sum_{n=1}^{\infty} z^{n} \log a_{n}$ is analytic in the unit disc, and (c) $\Phi(z)$ has the factorization (unique if $a_{1} \neq 1$ )

for some $p(z) \in P$.

$$
\Phi(z)=\left(z /(1-z)^{2}\right) p(z) \log a_{1}
$$

Proof. If $f \in P^{*}$, then all $a_{n} \neq 0$ if $a_{1} \neq 0$ and $\log a_{n}=O\left(n^{2}\right)$ by Theorem 1.8(e), so $\Phi(z) \equiv \sum_{n=1}^{\infty} z^{n} \log a_{n}$ is analytic. But we can compute $\Phi(z)$ from the representation formula in Theorem 1.9, and we find

$$
\begin{aligned}
\Phi(z)= & \sum_{n=1}^{\infty} z^{n} \log a_{n}=\log a_{1} \sum_{n=1}^{\infty} n z^{n} \\
& -\int_{-\pi}^{\pi} \frac{1}{\left(1-e^{-i \theta}\right)^{2}}\left\{e^{-i \theta} \sum_{n=1}^{\infty}\left(z e^{i \theta}\right)^{n}+e^{-i \theta} \sum_{n=1}^{\infty}(n-1) z^{n}-\sum_{n=1}^{\infty} n z^{n}\right\} d \mu(\theta) \\
= & \frac{z}{(1-z)^{2}} \log a_{1}-\int_{-\pi}^{\pi} \frac{z^{2} e^{i \theta}}{\left(1-z e^{i \theta}\right)(1-z)^{2}} d \mu(\theta) \\
= & \frac{z}{(1-z)^{2}}\left\{\log a_{1}-\int_{-\pi}^{\pi} \frac{z e^{i \theta}}{\left(1-z e^{i \theta}\right)} d \mu(\theta)\right\},
\end{aligned}
$$

where $d \mu(\theta)$ is a uniquely determined nonnegative measure of (finite) total mass $-2 \log a_{1} \geqq 0$. Let

so that in either case

$$
\begin{aligned}
d \nu(\theta) & \equiv d \mu(\theta) /-2 \log a_{1} & & \text { if } 0<a_{1}<1, \\
& \equiv d \theta / 2 \pi & & \text { if } a_{1}=1
\end{aligned}
$$

$$
\Phi(z)=\frac{z \log a_{1}}{(1-z)^{2}} \int_{-\pi}^{\pi} \frac{1+z e^{i \theta}}{1-z e^{i \theta}} d \nu(\theta)=\frac{z}{(1-z)^{2}} p(z) \log a_{1},
$$

where

$$
p(z) \equiv \int_{-\pi}^{\pi} \frac{1+z e^{i \theta}}{1-z e^{i \theta}} d \nu(\theta) \in P .
$$

The converse follows immediately from Theorem 1.9. Q.E.D. 
We can restate this in the language of measures as

COROLlaRY 1.15. Let $d \mu$ be a probability measure on $[-\pi, \pi)$ with mass center $C(d \mu)=a_{1}=\int_{-\pi}^{\pi} e^{i \theta} d \mu(\theta)>0$. Then $d \mu$ is strongly infinitely divisible if and only if (a) all higher moments $a_{n} \equiv \int_{-\pi}^{\pi} e^{i n \theta} d \mu(\theta) \neq 0$, (b) the generating function $F(z) \equiv$ $\sum_{n=1}^{\infty} z^{n} \log a_{n}$ is analytic in the unit disc, and (c) either $a_{1}=1$ or, if not,

$$
\left((z-1)^{2} / z \log a_{1}\right) F(z) \in P .
$$

Finally, we wish to discuss the interpolation problem for infinitely divisible positive definite (-) sequences, i.e., given such a sequence $\left\{a_{n}\right\}_{n=0}^{\infty} \geq 0(-), a_{0}=1$, to find if possible an infinitely divisible characteristic function $\phi$ such that $\phi(n)=a_{n}$ for all $n=0,1,2, \ldots$ We recall that a characteristic function is a uniformly continuous function $\phi: R \rightarrow C$ such that $\phi(0)=1$ and $K(x, y) \equiv \phi(x-y) \geq 0$ on $C_{0}(R)$. The characteristic function $\phi$ is said to be infinitely divisible if the kernel $K(x, y)$ is an infinitely divisible kernel [5]. Such functions have long been of importance in probability theory and are discussed thoroughly with our methods in [4].

This problem can be solved immediately using the facts we have already, but perhaps we should first remark that the interpolation problem for sequences $\left\{a_{n}\right\}_{n=0}^{\infty}, a_{0}=1$, which are just positive definite $(-)$ is trivial once one has the Herglotz representation formula of Theorem 1.2. Indeed, if $d \mu$ is the (unique) probability measure such that $a_{n}=\int_{-\pi}^{\pi} e^{i n \theta} d \mu(\theta)$ for all $n=0,1,2, \ldots$, then the characteristic function $\phi(x) \equiv \int_{-\pi}^{\pi} e^{i x \theta} d \mu(\theta)$ solves the problem.

The interpolation problem for an infinitely divisible positive definite $(-)$ sequence $\left\{a_{n}\right\}_{n=0}^{\infty}, a_{0}=1, a_{1} \neq 0$, is no less trivial given Theorem 1.9, for if $d \mu$ is the (unique) representing measure guaranteed by that theorem, we may take $\phi(x)$ $\equiv e^{f(x)}$, where

$$
\begin{aligned}
& f(x) \equiv x \log a_{1}-\int_{-\pi}^{\pi} \frac{e^{i(x-1) \theta}+(x-1) e^{-i \theta}-x}{\left(1-e^{-i \theta}\right)^{2}} d \mu(\theta) \quad \text { if } x \geqq 0, \\
& \equiv-x \overline{\log a_{1}}-\int_{-\pi}^{\pi} \frac{e^{i(x+1) \theta}-(x+1) e^{i \theta}+x}{\left(1-e^{i \theta}\right)^{2}} d \mu(\theta) \quad \text { if } x<0 .
\end{aligned}
$$

One checks readily that because $\int_{-\pi}^{\pi} d \mu(\theta)=-2 \operatorname{Re} \log a_{1}, f(x)$ is not only continuous, but actually $f \in C^{\infty}(\boldsymbol{R})$ and

$$
f^{\prime \prime}(x)=-\frac{1}{4} \int_{-\pi}^{\pi} e^{i x \theta}\left(\frac{\theta}{\sin (\theta / 2)}\right)^{2} d \mu(\theta) .
$$

Thus, $H(x, y) \equiv-f^{\prime \prime}(x-y) \geq 0$ on $C_{0}(\boldsymbol{R})$. But $\bar{f}(-x)=f(x)$ and $f(0)=0$ so we know [4, Theorem 3.4] that $\phi(x)=e^{f(x)}$ is an infinitely divisible characteristic function which, by construction, interpolates the given infinitely divisible positive definite sequence $\left\{a_{n}\right\}_{n=0}^{\infty}$. It is well to note, however, that if $\alpha>0$ is not an integer, then $\phi^{\alpha}(x)$ need not interpolate $\left\{a_{n}^{\alpha}\right\}_{n=0}^{\infty}$, although this interpolation problem does have some (other) solution. 
Because an infinitely divisible characteristic function can have no real zeros [4, Lemma 3.2], the interpolation problem cannot be solved if the infinitely divisible sequence $\left\{a_{n}\right\}_{n=0}^{\infty} \succeq 0(-)$ has any zero terms. We have then proved the

THEOREM 1.16. Let $\left\{a_{n}\right\}_{n=0}^{\infty}, a_{0}=1$, be an infinitely divisible positive definite (-) sequence. There exists an infinitely divisible characteristic function $\phi(x)$ with $\phi(n)=a_{n}$, $n=0,1,2, \ldots$, if and only if $a_{1} \neq 0$.

If a finite sequence $\left\{a_{n}\right\}_{n=0}^{N}, a_{0}=1$, is given which is such that $A_{N} \equiv\left(a_{i-j}\right)_{i, j=0}^{N} \succeq 0$, $a_{-k} \equiv \bar{a}_{k}$, then it is trivial that the periodic sequence $\left\{a_{n}^{\prime}\right\}_{n=0}^{\infty}, a_{n}^{\prime} \equiv a_{n \bmod N}$, is positive definite (-). If $A_{N}$ is infinitely divisible, then so is $\left\{a_{n}^{\prime}\right\}_{n=0}^{\infty}$. Thus, the interpolation problem for finite sequences is readily reduced to the cases treated above.

2. Hankel forms. If $\left\{a_{n}\right\}_{n=0}^{\infty} \subset \boldsymbol{C}$ is a sequence of complex numbers, we shall say that $\left\{a_{n}\right\}_{n=0}^{\infty}$ is a positive definite $(+)$ sequence and write $\left\{a_{n}\right\}_{n=0}^{\infty} \succeq 0(+)$ if and only if the Hankel matrix $A \equiv\left(a_{i+j}\right)_{i, j=0}^{\infty} \geq 0$. Such sequences are of importance in analysis because of their intimate connection with the algebraic moment problems of Hamburger, Stieltjes, and Hausdorff [7, pp. 4, 5, 8]. We begin by defining the difference sequences

$$
\left\{\Delta_{N} a_{n}\right\}_{n=0}^{\infty} \equiv\left\{\sum_{k=0}^{N}(-1)^{k}\left(\begin{array}{l}
N \\
k
\end{array}\right) a_{n+N-k}\right\}_{n=0}^{\infty}
$$

for any $N=0,1,2, \ldots$ and by stating some of the well-known (vid. [3, pp. 52-53]) fundamental properties of positive definite $(+)$ sequences as

THEOREM 2.1. Let $\left\{a_{n}\right\}_{n=0}^{\infty} \succeq 0(+)$. Then (a) all even terms $a_{2 n} \geqq 0$; (b) all $a_{n} \in \boldsymbol{R}$; (c) if $a_{0}=0$, then all $a_{n} \equiv 0$; (d) if some $a_{2 N}=0, N \geqq 1$, then $a_{n} \equiv 0$ for all $n=1,2,3$, ...; (e) if $\left\{b_{n}\right\}_{n=0}^{\infty} \succeq 0(+)$, then $\left\{a_{n} b_{n}\right\}_{n=0}^{\infty} \geq 0$ (+); (f) $\left\{\Delta_{2 N} a_{n}\right\}_{n=0}^{\infty} \geq 0(+)$ for all $N=0,1,2,3, \ldots$, and so $\Delta_{2 N} a_{2 n} \geqq 0$ for all $N, n=0,1,2, \ldots ;(\mathrm{g}) a_{0} a_{2} \geqq a_{1}^{2}$ and, more generally, $a_{2 i} a_{2 j} \geqq a_{i+j}^{2}$ for all $i, j \geqq 0$; and (h) if $a_{0} a_{2}=a_{1}^{2}$, then $a_{0}^{n-1} a_{n} \equiv a_{1}^{n}$ for all $n=1,2,3, \ldots$.

There will be, therefore, no loss of generality to assume in the following that $a_{0}=1$ and that all $a_{n} \in \boldsymbol{R}$. The fundamental representation formula for positive definite $(+)$ sequences is given by the classical theorem of Hamburger:

TheOREM 2.2 [7, ChAPTER II]. Let $\left\{a_{n}\right\}_{n=0}^{\infty} \subset \boldsymbol{R}, a_{0}=1$. Then $\left\{a_{n}\right\}_{n=0}^{\infty} \geq 0(+)$ if and only if $a_{n}=\int_{-\infty}^{\infty} x^{n} d \mu(x), n=0,1,2, \ldots$, for some probability measure $d \mu$ on $\boldsymbol{R}$.

The representing measure $d \mu$ for such a moment sequence is not in general unique. We introduce the notion of infinite divisibility of a moment sequence according to the following

Definition. The sequence $\left\{a_{n}\right\}_{n=0}^{\infty} \succeq 0(+)$ is said to be infinitely divisible if and only if the associated Hankel matrix $A \equiv\left(a_{i+j}\right)_{i, j=0}^{\infty}$ is infinitely divisible. 
Because of Theorem 2.1(b), we see that if $\left\{a_{n}\right\}_{n=0}^{\infty} \geq 0(+)$ is infinitely divisible, then necessarily all $a_{n} \geqq 0$ since, in particular, all the terms of $\left\{a_{n}^{1 / 2}\right\}_{n=0}^{\infty} \geq 0(+)$ must be real. Let the sequence $\left\{\mu_{n}\right\}_{n=0}^{\infty}$ be defined by

$$
\begin{aligned}
\mu_{n} \equiv 1 & \text { if } a_{n} \neq 0 \\
\equiv 0 & \text { if } a_{n}=0
\end{aligned}
$$

If $a_{2} \neq 0$ then all even terms $a_{2 n} \neq 0$, so if $\left\{a_{n}\right\}_{n=0}^{\infty} \geq 0(+)$ is infinitely divisible it is necessary [5, Theorem 1.14] for all $k=1,2,3, \ldots$ that the incidence matrix

$$
M\left(\begin{array}{lll}
a_{0} & a_{1} & a_{2 k} \\
a_{1} & a_{2} & a_{2 k+1} \\
a_{2 k} & a_{2 k+1} & a_{4 k}
\end{array}\right) \equiv\left(\begin{array}{lll}
\mu_{0} & \mu_{1} & \mu_{2 k} \\
\mu_{1} & \mu_{2} & \mu_{2 k+1} \\
\mu_{2 k} & \mu_{2 k+1} & \mu_{4 k}
\end{array}\right)=\left(\begin{array}{lll}
1 & \mu_{1} & 1 \\
\mu_{1} & 1 & \mu_{2 k+1} \\
1 & \mu_{2 k+1} & 1
\end{array}\right) \geq 0 .
$$

But then $\mu_{1}$ and $\mu_{2 k+1}$ must be 0 or 1 together, so we have proved the following

THEOREM 2.3. Let $\left\{a_{n}\right\}_{n=0}^{\infty} \geq 0(+)$ be infinitely divisible, $a_{0}=1$. Then either (a) $a_{2}=0$, in which case all $a_{n} \equiv 0, n=1,2,3, \ldots$, or (b) $a_{1}>0$, in which case all $a_{n}>0, n=0,1,2, \ldots$, or (c) $a_{1}=0, a_{2} \neq 0$, in which case all $a_{2 n}>0, a_{2 n+1} \equiv 0$, $n=0,1,2, \ldots$ These statements are mutually exclusive and exhaust all possibilities.

Because of this and the following two results, we may assume without loss of generality in the following that the terms of an infinitely divisible positive definite $(+)$ sequence are all strictly positive.

Proposition 2.4. The sequence $\left\{b_{n}\right\}_{n=0}^{\infty} \equiv E_{2}\left\{a_{n}\right\}_{n=0}^{\infty}$ is positive definite $(+)$ if and only if both $\left\{a_{n}\right\}_{n=0}^{\infty}$ and $\left\{a_{n+1}\right\}_{n=0}^{\infty}$ are positive definite $(+)$ sequences. We recall that $b_{2 n} \equiv a_{n}$ and $b_{2 n+1} \equiv 0$ for all $n=0,1,2, \ldots$

Proof. This follows immediately from the identity

$$
\sum_{i, j=0} b_{i+j} x_{i} \bar{x}_{j} \equiv \sum_{i, j=0} a_{i+j} x_{2 i} \bar{x}_{2 j}+\sum_{i, j=0} a_{i+j+1} x_{2 i+1} \bar{x}_{2 j+1}
$$

COROLlary 2.5. The sequence $\left\{a_{n}\right\}_{n=0}^{\infty}, a_{0}=1, a_{1}=0, a_{2} \neq 0$ is an infinitely divisible positive definite $(+)$ sequence if and only if both $\left\{a_{2 n}\right\}_{n=0}^{\infty}$ and $\left\{a_{2 n+2}\right\}_{n=0}^{\infty}$ are infinitely divisible positive definite $(+)$ sequences with strictly positive terms.

Thus, an infinitely divisible positive definite $(+)$ sequence $\left\{a_{n}\right\}_{n=0}^{\infty}, a_{0}=1$, is either the trivial sequence $\left\{\delta_{n 0}\right\}_{n=0}^{\infty}$, or it has all positive terms, or it is a 2-sequence whose fundamental sequence is, as we shall see later, an infinitely divisible Stieltjes moment sequence. Reserving the last case for separate treatment later, we can make a direct application of [5, Corollary 1.6] to obtain the

THEOREM 2.6. Let $\left\{a_{n}\right\}_{n=0}^{\infty} \subset R, a_{0}=1, a_{1} \neq 0$. Then $\left\{a_{n}\right\}_{n=0}^{\infty}$ is an infinitely divisible positive definite $(+)$ sequence if and only if all $a_{n}>0$ and $B \equiv\left(\log a_{i+j}\right)_{i, j=0}^{\infty} \geq 0$ on $L$. 
Recall that whenever $\left\{a_{n}\right\}_{n=0}^{\infty} \geq 0(+), a_{0}=1$, then $a_{2} a_{1}^{-2} \geqq 1$ and if $a_{2} a_{1}^{-2}=1$, then $a_{n} \equiv a_{1}^{n}$ for all $n=0,1,2, \ldots$ This behavior of all positive definite $(+)$ sequences is reflected in the fundamental representation formula contained in

TheOREM 2.7. Let $\left\{a_{n}\right\}_{n=0}^{\infty} \subset \boldsymbol{R}, a_{0}=1, a_{1} \neq 0$. Then $\left\{a_{n}\right\}_{n=0}^{\infty}$ is an infinitely divisible positive definite $(+)$ sequence if and only if all $a_{n}>0, n=0,1,2, \ldots$, and there exists a nonnegative measure $d \mu$ on $\boldsymbol{R}$ with $\int_{-\infty}^{\infty} d \mu(x)=\log \left(a_{2} / a_{1}^{2}\right)$ such that

(2.A) $\log a_{n}=n \log a_{1}+\int_{-\infty}^{\infty} \frac{x^{n}-n x+n-1}{(1-x)^{2}} d \mu(x), \quad n=0,1,2, \ldots$

Proof. If $\left\{a_{n}\right\}_{n=0}^{\infty} \geq 0(+)$ is infinitely divisible, then since $a_{1} \neq 0$ we know all $a_{n}>0$ and that $B=\left(\log a_{i+j}\right)_{i, j=0}^{\infty} \succeq 0$ on $L$. But then [5, Theorem 1.10] we must have

$$
\Delta B=\left(\log \left(a_{i+j} a_{i+j+2} / a_{i+j+1}^{2}\right)\right)_{i, j=0}^{\infty} \succeq 0
$$

so that

$$
\left\{\log \left(a_{n} a_{n+2} / a_{n+1}^{2}\right)\right\}_{n=0}^{\infty} \geq 0(+) .
$$

By Theorem 2.2 there is some nonnegative measure $d \mu$ on $\boldsymbol{R}$ with

$$
\int_{-\infty}^{\infty} d \mu(x)=\log \frac{a_{0} a_{2}}{a_{1}^{2}}=\log \frac{a_{2}}{a_{1}^{2}}
$$

such that

$$
\log \frac{a_{n} a_{n+2}}{a_{n+1}^{2}}=\int_{-\infty}^{\infty} x^{n} d \mu(x), \quad n=0,1,2, \ldots
$$

Let $b_{n} \equiv a_{n} a_{n+2} a_{n+1}^{-2}$, so that $a_{n+2}=a_{1}^{n+2} b_{n} b_{n-1}^{2} b_{n-2}^{3} \cdots b_{1}^{n} b_{0}^{n+1}, n=0,1,2, \ldots$ But then

$$
\begin{aligned}
\log a_{n+2} & =(n+2) \log a_{1}+\sum_{k=0}^{n}(n+1-k) \log b_{k} \\
& =(n+2) \log a_{1}+\int_{-\infty}^{\infty} \sum_{k=0}^{n}(n+1-k) x^{k} d \mu(x) \\
& =(n+2) \log a_{1}+\int_{-\infty}^{\infty} \frac{x^{n+2}-(n+2) x+(n+1)}{(1-x)^{2}} d \mu(x), \quad n=0,1,2, \ldots
\end{aligned}
$$

Since this formula also holds for $n=-2,-1,(2 . \mathrm{A})$ is proved.

Conversely, if (2.A) holds, then $\Delta B=\left(c_{i+j}\right)_{i, j=0}^{\infty}$ where $c_{n}=\int_{-\infty}^{\infty} x^{n} d \mu(x)$, so $\Delta B \geq 0$ and hence $B \geq 0$ on $L$. By Theorem $2.6,\left\{a_{n}\right\}_{n=0}^{\infty}$ must be infinitely divisible. Q.E.D.

We now wish to consider the problem of identifying the infinitely divisible sequences associated with the special moment problems of Stieltjes and Hausdorff, so we shall need the classical representation theorems in each case.

THEOREM 2.8 [7], [8]. Let $\left\{a_{n}\right\}_{n=0}^{\infty} \succeq 0(+), a_{0}=1$, so that $a_{n}=\int_{-\infty}^{\infty} x^{n} d \mu(x)$ for some probability measure $d \mu$ on $\boldsymbol{R}$. In order that, in addition, $d \mu$ should have support only 
on (a) $[0, \infty),(b)[-1,1]$, or (c) $[0,1]$, respectively, it is necessary and sufficient that $\left(\mathrm{a}^{\prime}\right)\left\{a_{n+1}\right\}_{n=0}^{\infty} \geq 0(+),\left(\mathrm{b}^{\prime}\right)\left\{a_{n}-a_{n+2}\right\}_{n=0}^{\infty} \geq 0(+)$, or $\left(\mathrm{c}^{\prime}\right)\left\{a_{n+1}\right\}_{n=0}^{\infty},\left\{a_{n}-a_{n+1}\right\}_{n=0}^{\infty}$ $=\left\{-\Delta_{1} a_{n}\right\}_{n=0}^{\infty}$, and $\left\{a_{n+1}-a_{n+2}\right\}_{n=0}^{\infty}=\left\{-\Delta_{1} a_{n+1}\right\}_{n=0}^{\infty}$ are all $0 \geq(+)$, respectively. In cases (b) and (c), the measure $d \mu$ is unique.

With each moment problem there is a natural notion of infinite divisibility, viz., that all the sequences in the conditions $\left(a^{\prime}\right),\left(b^{\prime}\right),\left(c^{\prime}\right)$ as well as the original sequence $\left\{a_{n}\right\}_{n=0}^{\infty}$ itself should remain positive definite $(+)$ if $a_{n}$ is replaced by $a_{n}^{\alpha}$ for any $\alpha>0$. Since these are all special cases of the infinitely divisible Hamburger problem, all our previous results 2.1-2.7 remain valid, but more can be said in each case. In particular, in cases $\left(\mathrm{a}^{\prime}\right)$ and $\left(\mathrm{c}^{\prime}\right)$ it is clear that $a_{1}=0$ implies $a_{n} \equiv 0, n \geqq 1$. Let us consider the analogues of Theorem 2.7 in each case.

THEOREM 2.9. Let $\left\{a_{n}\right\}_{n=0}^{\infty} \subset \boldsymbol{R}, a_{0}=1, a_{1} \neq 0$. Then $\left\{a_{n}\right\}_{n=0}^{\infty}$ is an infinitely divisible positive definite $(+)$ sequence in the sense of part $\left(\mathrm{a}^{\prime}\right),\left(\mathrm{b}^{\prime}\right)$, or $\left(\mathrm{c}^{\prime}\right)$ of Theorem 2.8 if and only if in each case all $a_{n}>0$ and there exists, respectively, a nonnegative measure $d \mu$ on $\boldsymbol{R}$ such that for all $n=0,1,2, \ldots$

(a) $\log a_{n}=n \log a_{1}+\int_{0}^{\infty} \frac{x^{n}-n x+n-1}{(1-x)^{2}} d \mu(x), \quad \int_{0}^{\infty} d \mu(x)=\log \frac{a_{2}}{a_{1}^{2}} \geqq 0$,

(b) $\log a_{n}=-\int_{-1}^{1} \frac{1-x^{n}}{1-x} d \mu(x), \quad \int_{-1}^{1} d \mu(x)=-\log a_{1} \geqq 0$,

or,

$$
\log a_{n}=-\int_{0}^{1} \frac{1-x^{n}}{1-x} d \mu(x), \quad \int_{0}^{1} d \mu(x)=-\log a_{1} \geqq 0 .
$$

In cases (b) and (c) the measure $d \mu$ is unique.

Proof. (a) follows by an argument similar to that of Theorem 2.7, while if one notices that condition ( $\left.b^{\prime}\right)$ of Theorem 2.8 is equivalent to the pair of conditions $\left\{a_{n} \pm a_{n+1}\right\}_{n=0}^{\infty} \geq 0(+)$, then part (b) can be handled in a way similar to the proof of part (c), which we now give. The following six statements are easily seen to be sequentially equivalent:

(i) $\left\{a_{n}^{\alpha}\right\},\left\{a_{n+1}^{\alpha}\right\},\left\{a_{n}^{\alpha}-a_{n+1}^{\alpha}\right\},\left\{a_{n+1}^{\alpha}-a_{n+2}^{\alpha}\right\}$ are all $\geq 0(+)$ for all $\alpha>0$;

(ii) $\left\{\log a_{n}\right\},\left\{\log a_{n+1}\right\} \succeq 0(+)$ on $L$, while $\left\{\log \left(a_{n} / a_{n+1}\right)\right\},\left\{\log \left(a_{n+1} / a_{n+2}\right)\right\}$ $\geq 0(+)$;

(iii) $\left\{\log \left(a_{n} a_{n+2} / a_{n+1}^{2}\right)\right\},\left\{\log \left(a_{n+1} a_{n+3} / a_{n+2}^{2}\right)\right\},\left\{\log \left(a_{n} / a_{n+1}\right)\right\},\left\{\log \left(a_{n+1} / a_{n+2}\right)\right\}$ are all $\geq 0(+)$;

(iv) $\left\{\log \left(a_{n} / a_{n+1}\right)\right\},\left\{\log \left(a_{n+1} / a_{n+2}\right)\right\}, \quad\left\{\log \left(a_{n} / a_{n+1}\right)-\log \left(a_{n+1} / a_{n+2}\right)\right\}$, and $\left\{\log \left(a_{n+1} / a_{n+2}\right)-\log \left(a_{n+2} / a_{n+3}\right)\right\}$ are all $\geq 0(+)$, i.e., the sequence

$$
\left\{\log \left(a_{n} / a_{n+1}\right)\right\}_{n=0}^{\infty}
$$

itself satisfies the conditions of $\left(\mathrm{c}^{\prime}\right)$ of Theorem 2.8 . 
(v) There exists a (unique) nonnegative measure $d \mu$ on $[0,1]$ with $\int_{0}^{1} d \mu(x)$ $=\log \left(a_{0} / a_{1}\right)=-\log a_{1}$ such that $\log \left(a_{n} / a_{n+1}\right)=\int_{0}^{1} x^{n} d \mu(x)$ for all $n=0,1,2, \ldots$;

(vi) $\log a_{n}=-\int_{0}^{1}\left(\left(1-x^{n}\right) /(1-x)\right) d \mu(x), n=0,1, \ldots$, for a (unique) $d \mu \geqq 0$ such that $\int_{0}^{1} d \mu(x)=-\log a_{1}$. Q.E.D.

Sequences which satisfy the equivalent conditions (c), $\left(\mathrm{c}^{\prime}\right)$ of Theorem 2.8 are called completely monotonic. It is well known [7, p. 87] that $\left\{a_{n}\right\}_{n=0}^{\infty}$ is completely monotonic if and only if $(-1)^{N} \Delta_{N} \mu_{n} \geqq 0$ for all $n, N=0,1,2, \ldots$ If both $\left\{a_{n}\right\}_{n=0}^{\infty}$ and $\left\{b_{n}\right\}_{n=0}^{\infty}$ are completely monotonic sequences, then so is $\left\{a_{n} b_{n}\right\}_{n=0}^{\infty}$, since the definition and Theorem 2.1(e) guarantee that each of the sequences $\left\{a_{n} b_{n}\right\}_{n=0}^{\infty}$, $\left\{a_{n+1} b_{n+1}\right\}_{n=0}^{\infty}, \quad\left\{a_{n} b_{n}-a_{n+1} b_{n+1}\right\}_{n=0}^{\infty}=\left\{a_{n}\left(b_{n}-b_{n+1}\right)+b_{n+1}\left(a_{n}-a_{n+1}\right)\right\}_{n=0}^{\infty}$, and $\left\{a_{n+1} b_{n+1}-a_{n+2} b_{n+2}\right\}_{n=0}^{\infty}=\left\{a_{n+1}\left(b_{n+1}-b_{n+2}\right)+b_{n+2}\left(a_{n+1}-a_{n+2}\right)\right\}_{n=0}^{\infty}$ is positive definite $(+)$.

If $a_{n}=\int_{0}^{1} x^{n} d \alpha(x), b_{n}=\int_{0}^{1} x^{n} d \beta(x)$, then $a_{n} b_{n}=\int_{0}^{1} x^{n}(d \alpha * d \beta)(x)$, where $d \alpha * d \beta$ is the multiplicative convolution in the (multiplicative) semigroup [0,1]. That is, $(d \alpha * d \beta)$ has the distribution function

$$
(\alpha * \beta)(x)=\int_{x}^{1} d \alpha * d \beta=\int_{x}^{1} \alpha\left(\frac{x}{y}\right) d \beta(y)=\int_{x}^{1} \beta\left(\frac{x}{y}\right) d \alpha(y)
$$

if $\alpha(x) \equiv \int_{x}^{1} d \alpha$ and $\beta(x) \equiv \int_{x}^{1} d \beta$ are the respective distribution functions of $d \alpha$ and $d \beta$.

We have immediately the

THEOREM 2.10. Let $d \mu$ be a measure on $[0,1]$. Then $d \mu$ is a (strongly) infinitely divisible probability measure if and only if the sequence $\left\{a_{n}\right\}_{n=0}^{\infty}=\left\{\int_{0}^{1} x^{n} d \mu(x)\right\}_{n=0}^{\infty}$ is an infinitely divisible completely monotonic sequence with $a_{0}=1$.

Notice that because the moments $\int_{0}^{1} x^{n} d \mu(x)$ are nonnegative and have unique nonnegative $N$ th roots, every infinitely divisible measure on $[0,1]$ (under multiplication) is also strongly infinitely divisible.

Because $a_{1}=0$ in a completely monotonic sequence $\left\{a_{n}\right\}_{n=0}^{\infty}$ implies $a_{n} \equiv 0$, $n=2,3,4, \ldots$, we see that if $d \mu$ is an infinitely divisible probability measure on $[0,1]$, either $d \mu=\delta(0)=$ point mass one at 0 , or all moments $a_{n}=\int_{0}^{1} x^{n} d \mu(x)>0$. We therefore have from part (iv) of the proof of Theorem 2.9 the

THEOREM 2.11. Suppose $d \mu$ is a probability measure on $[0,1], d \mu \neq \delta(0)$. Then $d \mu$ is (strongly) infinitely divisible if and only if the sequence $\left\{\log a_{n} a_{n+1}^{-1}\right\}_{n=0}^{\infty}$ is completely monotonic, where $a_{n} \equiv \int_{0}^{1} x^{n} d \mu(x)$.

Analytic functions with nonnegative real part in the unit disc were closely related to the trigonometric moment problem, so it is not surprising that there is a natural class of functions associated with the algebraic moment problem. We consider the case of a compact interval, which we shall take to be $[-1,1]$.

DEFINITION. The class of normalized positive imaginary part functions will be denoted by $\mathrm{PI} \equiv\{f(z)$ analytic in the upper half plane $\operatorname{Im}(z)>0 \mid f(z)$ is real valued and analytic on $(-1,1), f(0)=0, f^{\prime}(0)=1, \operatorname{Im} f(z) \geqq 0$ when $\left.\operatorname{Im}(z)>0\right\}$. 
It is known [6, p. 242] that $f \in \mathrm{PI}$ if and only if for some probability measure $d \mu$ on $[-1,1], f(z)=\int_{-1}^{1} z(1-z t)^{-1} d \mu(t)$ for all $z$ in the complex plane with the two half lines $[1, \infty)$ and $(-\infty,-1]$ deleted. But then if $-1<x<1$,

$$
f(x)=\sum_{n=0}^{\infty} x^{n+1} \int_{-1}^{1} t^{n} d \mu(t) \equiv \sum_{n=0}^{\infty} a_{n} x^{n+1},
$$

i.e., the coefficients of the power series for $f(z)$ in $\{|z|<1\}$ are a moment sequence on $[-1,1]$ and conversely. If this moment sequence is infinitely divisible, we shall say that $f(z)$ is infinitely divisible, and we have

$$
f_{\alpha}(z) \equiv \sum_{n=0}^{\infty} a_{n}^{\alpha} z^{n+1}=\int_{-1}^{1} \frac{z d \mu_{\alpha}(t)}{1-z t} \in \mathrm{PI}
$$

for some family $\left\{d \mu_{t}\right\}_{t>0}$ of probability measures on $[-1,1]$ for all $\alpha>0$. If $a_{1} \neq 0$, then all $a_{n} \neq 0$ and we can compute the infinitesimal generating function

$$
\begin{aligned}
\Phi(z) & \left.\equiv \frac{d}{d \alpha} f_{\alpha}(z)\right|_{\alpha=0}=\sum_{n=0}^{\infty} z^{n+1} \log a_{n} \\
& =-\sum_{n=0}^{\infty} z^{n+1} \int_{-1}^{1} \frac{1-t^{n}}{1-t} d \beta(t)=\left(\log a_{1}\right) \frac{z}{1-z} \int_{-1}^{1} \frac{z d \nu(t)}{1-z t}
\end{aligned}
$$

for some (unique) probability measure $d \nu(t) \equiv-\left(\log a_{1}\right)^{-1} d \beta(t)$ if $a_{1} \neq 1$. We state this formally as

TheOREM 2.12. Let $f(z) \in \mathrm{PI}, f^{\prime \prime}(0) \neq 0, f(z)=\sum_{n=0}^{\infty} a_{n} z^{n+1}$ for $|z|<1$. Then $f(z)$ is infinitely divisible if and only if all $a_{n}>0$ and

$$
\Phi(z)=\sum_{n=0}^{\infty} z^{n+1} \log a_{n}=\frac{z}{1-z} q(z) \log a_{1}
$$

for some $q(z) \in \mathrm{PI}$ which is uniquely determined if $f^{\prime \prime}(0) \neq 2$.

Finally, we wish to discuss the interpolation problem for infinitely divisible completely monotonic sequences, i.e., given such a sequence $\left\{a_{n}\right\}_{n=0}^{\infty}$ to find if possible an infinitely divisible completely monotonic function $\phi$ such that $\phi(n)=a_{n}$ for all $n=0,1,2, \ldots$ We recall that a completely monotonic function is a continuously differentiable function $\phi: \boldsymbol{R}^{+} \rightarrow \boldsymbol{R}^{+}$such that the kernels $K_{1}(x, y) \equiv \phi(x+y)$ and $K_{2}(x, y) \equiv-\phi^{\prime}(x+y)$ are positive semidefinite on $C_{0}\left(\boldsymbol{R}^{+}\right)$. The completely monotonic function $\phi$ is said to be infinitely divisible if $\phi^{\alpha}$ is a completely monotonic function for all $\alpha>0$, where the positive root is always chosen. These functions are discussed with our methods in [4], where we find that $\phi$ is an infinitely divisible completely monotonic function with $\phi\left(0^{+}\right)=1$ if and only if $\phi(x)>0$ for all $x>0$ and

$$
\log \phi(x)=-\int_{0}^{\infty} \frac{1-e^{-x t}}{1-e^{-t}} d \nu(t)
$$


for some (unique) nonnegative measure $d v$ such that the integral is convergent for all $x>0$.

We should also recall the classical theorem of Bernstein which says that $\phi$ is a completely monotonic function with $\phi\left(0^{+}\right)=1$ if and only if there exists a (unique) probability measure $d \mu$ such that

$$
\phi(x)=\int_{0}^{\infty} e^{-x t} d \mu(t)
$$

for all $x>0$. In particular, $\phi$ is a bounded analytic function in the right half plane. Of course, any such completely monotonic function always gives rise to a completely monotonic sequence $\{\phi(n)\}_{n=0}^{\infty}$ which will be infinitely divisible if the function is. We wish to consider the converse problem.

It is well known that the interpolation problem for the (ordinary) completely monotonic sequence $\left\{a_{n}\right\}_{n=0}^{\infty}=\left\{\int_{0}^{1} s^{n} d v(s)\right\}_{n=0}^{\infty}$ has a solution if and only if the (unique) associated measure $d \nu(s)$ has no point mass at $s=0$, and the reason for this is very simple. If $\phi(x)=\int_{0}^{\infty} e^{-x t} d \mu(t)$ with $d \mu$ a positive finite measure, then

$$
\{\phi(n)\}_{n=0}^{\infty}=\left\{\int_{0}^{\infty} e^{-n t} d \mu(t)\right\} \equiv\left\{\int_{0}^{1} s^{n} d \nu(s)\right\}_{n=0}^{\infty},
$$

where $d \nu(s) \equiv-d \mu(-\log s) \geqq 0$ and $d \nu(0)=\lim _{T \rightarrow \infty} \int_{T}^{\infty} d \mu(t)=0$. Conversely, if $a_{n}=\int_{0}^{1} s^{n} d \nu(s)$, then if $d v(0)=0$ one could just change variables, letting $s=e^{-t}$, to find an interpolating function $\phi(x) \equiv \int_{0}^{\infty} e^{-x t} d \mu(t)$, where $d \mu(t) \equiv-d \nu\left(e^{-t}\right)$.

Definition. The completely monotonic sequence $\left\{a_{n}\right\}_{n=0}^{\infty}$ is said to be minimal if and only if the sequence $\left\{a_{n}\left(\exp -\varepsilon \delta_{n 0}\right)\right\}_{n=0}^{\infty}$ is not completely monotonic for any $\varepsilon>0$, i.e., if and only if the sequence fails to be completely monotonic whenever $a_{0}$ is diminished.

It is easy to show $[8$, p. 164$]$ that the completely monotonic sequence $\left\{a_{n}\right\}_{n=0}^{\infty}$ is minimal if and only if the associated measure $d \nu$ has no point mass at zero, so we see that there exists a completely monotonic function which interpolates a given completely monotonic sequence if and only if the sequence is minimal.

Now let $\left\{a_{n}\right\}_{n=0}^{\infty}$ be an infinitely divisible completely monotonic sequence. If it can be interpolated by an infinitely divisible completely monotonic function it can, in particular, be interpolated by a completely monotonic function and the sequence must therefore be minimal. Conversely, if the sequence is minimal, then each completely monotonic sequence $\left\{a_{n}^{1 / N}\right\}_{n=0}$ must also be minimal, $N=1,2,3, \ldots$, and so there exist completely monotonic functions $\phi_{N}$ which interpolate each of these sequences, i.e., $\phi_{N}(n)=a_{n}^{1 / N}$ for all $n=0,1,2, \ldots$ and all $N=1,2,3, \ldots$ But the product of completely monotonic functions is completely monotonic [4], so the functions $\left(\phi_{N}\right)^{N}$ are also completely monotonic and $\left(\phi_{N}\right)^{N}(n)=a_{n}=\phi(n)$ for all $n=0,1,2, \ldots$ It follows from a familiar theorem of Blaschke [2, p. 255] that two bounded analytic functions in the right half plane must be identical if they take the same values at the points $z=0,1,2, \ldots$, and hence $\left(\phi_{N}\right)^{N} \equiv \phi$ for all $N=1,2$, 
$3, \ldots$ Thus, $(\phi)^{1 / N} \equiv \phi_{N}$ is completely monotonic for all $N=1,2,3, \ldots$, and so $\phi$ is an infinitely divisible completely monotonic function. This completes the proof of

THEOREM 2.13. Let $\left\{a_{n}\right\}_{n=0}^{\infty}, a_{0}=1$, be an infinitely divisible completely monotonic sequence. There exists an infinitely divisible completely monotonic function $\phi$ with $\phi(n)=a_{n}, n=0,1,2, \ldots$, if and only if the sequence $\left\{a_{n}\right\}_{n=0}^{\infty}$ is minimal.

We see that an infinitely divisible completely monotonic sequence can be interpolated by an infinitely divisible completely monotonic function if and only if it can be interpolated by a completely monotonic function. It is easy to show that another equivalent condition is that the measure appearing in the logarithmic representation formula for an infinitely divisible completely monotonic sequence has no point mass at zero; this fact may be used [3, pp. 91-93] to give a direct proof of the above theorem which avoids the use of Blaschke's theorem.

ACKNOWLEDgements. The advice and encouragement of Professors C. Loewner and D. C. Spencer during this work are gratefully acknowledged, as is the support of the National Science Foundation through its Graduate Fellowship Program.

\section{BIBLIOGRAPHY}

1. G. Herglotz, Über Potenzreihen mit positivem reellen Teil im Einheitskreis, S.-B. Sächs. Akad. Wiss. Leipzig Math.-Natur. Kl. (Math.-Phys. Kl.) 63 (1911), 501-511.

2. E. Hille, Analytic function theory, Vol. II, Ginn, Boston, Mass., 1962.

3. R. A. Horn, Infinitely divisible matrices, kernels, and functions, Ph.D. Dissertation, Stanford Univ., 1967.

4. - On infinitely divisible matrices, kernels, and functions, Z. Wahrscheinlichkeitstheorie und Verw. Gebiete 8 (1967), 219-230.

5. - The theory of infinitely divisible matrices and kernels, Trans. Amer. Math. Soc. 136 (1969), 269-286.

6. A. Koranyi, Note on the theory of monotone operator functions, Acta Sci. Math. (Szeged) 16 (1955), 241-245.

7. J. A. Shohat and J. D. Tamarkin, The problem of moments, Math. Surveys No. 1, Amer. Math. Soc., Providence, R. I., 1943.

8. D. V. Widder, The Laplace transform, Princeton Univ. Press, Princeton, N. J., 1946.

STANFORD UNIVERSITY, STANFord, CALIForNia 